\title{
ANÁLISIS
}

\section{EL BIBLIOTECARIO INTEGRADO EN EL APRENDIZAJE} UNIVERSITARIO

\author{
Mercedes Caridad-Sebastián y Sara Martínez-Cardama
}
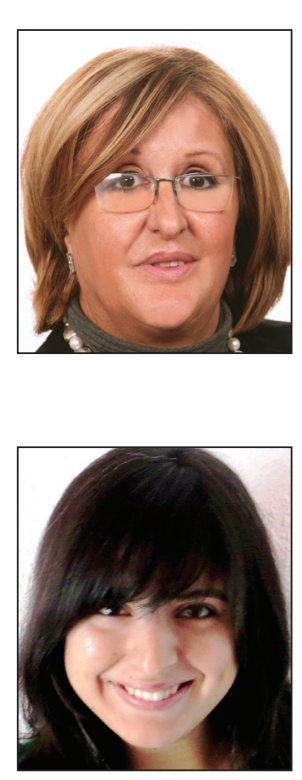

Mercedes Caridad-Sebastián es licenciada en ciencias de la información por la Universidad Complutense (1979), catedrática de la Universidad Carlos III de Madrid (UC3M), Departamento de Biblioteconomía y ha sido vicerrectora de Extensión Universitaria de la UC3M desde 1994 a 2007. Dirige el Instituto Agustín Millares de Documentación y Gestión de la Información. Tiene publicados numerosos libros y artículos en revistas nacionales e internacionales de prestigio reconocido en el sector.

http://orcid.org/0000-0001-7486-8353

Universidad Carlos III de Madrid C/ Madrid, 128. 28903 Getafe (Madrid), España mercedes@bib.uc3m.es

Sara Martínez-Cardama es investigadora del Programa de Formación de Profesorado Universitario (FPU) del Mo de Educación y docente en el Depto. de Biblioteconomía y Documentación de la Univ. Carlos III de Madrid. Diplomada y licenciada en documentación por la Univ. de A Coruña con Premio Nacional. Ha trabajado en diferentes organismos públicos como becaria, como el Congreso de los Diputados y el Senado de España. Entre sus líneas de investigación destacan la conceptualización y reformulación del espacio web e imagen digital de las bibliotecas universitarias. http://orcid.org/0000-0001-7035-5884

Universidad Carlos III de Madrid C/ Madrid, 128. 28903 Getafe (Madrid), España smarti1@bib.uc3m.es

\section{Resumen}

Visión general de las funciones educativas del bibliotecario universitario integrado (embedded librarian), basada en la colaboración y el establecimiento de relaciones con la comunidad académica. Concepto, funciones y modelos de integración en entornos de aprendizaje. Se concluye que las actividades de integración propuestas constituyen un buen modelo para sacar el máximo partido a la biblioteca dentro de la educación superior y legitima su participación en la actividad docente.

\section{Palabras clave}

Bibliotecas universitarias, Embedded librarians, Bibliotecarios integrados, Educación superior.

\section{Title: Embedded librarians in university education}

\section{Abstract}

Overview of educational roles of the embedded librarians, based on collaboration and building relationships with the faculty. The concept, functions and models of embedding librarians in learning environments are defined. It is concluded that embedding activities are a good model to empower the library in higher education and legitimate its participation in teaching activities.

\section{Keywords}

Academic libraries, Embedded librarians, Integration, Higher education.

Caridad-Sebastián, Mercedes; Martínez-Cardama, Sara (2013). “El bibliotecario integrado en el aprendizaje universitario". El profesional de la información, marzo-abril, v. 22, n. 2, pp. 149-154.

http://dx.doi.org/10.3145/epi.2013.mar.09 


\section{Introducción}

Las tecnologías de la información y comunicación (TIC) han supuesto un cambio para la profesión, y las unidades de información y los perfiles profesionales asociados han tenido que adaptarse a la nueva realidad.

Desde su aparición por primera vez en 2004 (Dewey, 2004), el término embedded librarianship o "biblioteconomía integrada" ${ }^{1}$ ha sido ampliamente tratado en la bibliografía aunque no bien acotado debido a la amplitud de campos y objetivos que trata (Drewes; Hoffman, 2010). Su aplicación en bibliotecas universitarias recoge la gran tradición de los servicios de referencia. La reinvención de su papel educativo a través del movimiento de alfabetización informacional (alfin) ha fortalecido la colaboración profesorado-biblioteca convirtiéndola en un elemento clave en el proceso docente (trascendiendo del papel de proveedor de material de apoyo a los estudiantes).

La biblioteca necesita una mayor integración para mejorar su imagen y la de la universidad, y para ello debe establecer colaboraciones con todos los agentes implicados en el proceso de enseñanza y aprendizaje (Pinto-Molina, 2011). Esta integración es cada vez más diversa desde la irrupción de las nuevas tecnologías y especialmente de los canales sociales o de la web 2.0, que han transformado nuestra manera de comunicarnos, y también de acercar la biblioteca a la comunidad académica.

\section{Antecedentes y concepto}

Dewey estableció en 2004 una de las primeras aproximaciones al concepto de "bibliotecario integrado" mediante un paralelismo con los llamados embedded journalists, los periodistas que acompañaron al ejército americano en las guerras de Afganistán e Irak.

Su aplicación al ámbito de la Documentación a tenido a veces un carácter difuso, y puede ser considerado como un término de moda o pasajero (buzz word) o un concepto que enmascara prácticas realizadas anteriormente. En su definición Dewey habla de: "integración más extensa en otro grupo para adquirir sus costumbres mediante la observación". Kvenild y Calkins (2011) definen este perfil como "aquellos que trabajan largos períodos de tiempo conjuntamente con grupos de carácter no bibliotecario". Shumaker lo define como aquel que enfoca su actividad basándose en las necesidades de un grupo reducido, compartiendo sus objetivos, métodos de trabajo, etc. La base de su definición es la construcción de relaciones (partnerships) con los usuarios. Es por tanto más relevante el concepto de colaboración que el de servicio.

También puede considerarse en contraposición o como ampliación de las actividades de referencia tradicionales (físicas o virtuales). Mientras éstas satisfacen demandas puntuales y el profesional referencista no precisa conocer al detalle los objetivos y proyectos del usuario, el integrado ha de comprender la misión general y los objetivos del grupo, su cultura de trabajo, creando vínculos y formando parte del mismo. Para ello puede o no desplazarse físicamente. Muchas descripciones del bibliotecario integrado trasladan su lugar de trabajo habitual fuera de la biblioteca, en una oficina satélite (Shumaker; Telley, 2009). Sin embargo la tecnología ha ampliado el concepto tradicional de biblioteconomía integrada y permite que este profesional, sin salir del lugar físico de la biblioteca, tenga una presencia similar. A pesar de que pueda parecer a priori más sencilla una comunicación cara a cara para lograr una integración total en el ambiente de trabajo, la experiencia muestra que a distancia es igualmente exitosa (Shumaker, 2012).

\section{El bibliotecario integrado ha de com- prender la misión general y objetivos del grupo, su cultura de trabajo y formar parte del mismo}

Los ejemplos de este perfil profesional son diversos, desde su integración en unidades de negocio de las empresas hasta en equipos interdisciplinares médicos, en los que muchos han liberado su carácter auxiliar para considerarse personal especializado que puede contribuir de manera vital en diferentes situaciones clínicas (Giuse, 1997).

Sin embargo donde más ha primado recientemente dicho concepto es en las bibliotecas universitarias. La mayoría de artículos lo identifican con la idea del "bibliotecario como formador" (especialmente en la educación a distancia) y como miembro de equipos de investigación (Torres-Salinas, 2011). Este estudio se centra en la primera opción.

El medio digital es cada vez más oportuno para este tipo de construcción de relaciones, desligándose así de la necesidad de ejercer su trabajo en un lugar físico determinado. Algunos autores llegan a afirmar que la biblioteconomía integrada constituye el principio del fin del la biblioteca como lugar físico (Matava; Coffey; Kushkowski, 2010). Este just be there implica que no importa el lugar, sino formar parte de la comunidad universitaria y no de un servicio en sí mismo (Covone; Lamm, 2010).

\section{El bibliotecario integrado en el proceso de aprendizaje. Aproximación y actividades principales}

La colaboración biblioteca-profesorado se ha venido fraguando desde los años 70, especialmente en Estados Unidos. Se inicia con la aparición de los bibliotecarios temáticos denominados liaison librarians, que en colaboración con los docentes realizaban una instrucción bibliotecaria tradicional para los estudiantes. Su labor ha evolucionado y actualmente realizan actividades que entrarían en las propias de bibliotecario integrado, como la creación de guías de recursos temáticos. Una muestra más de que la terminología necesita todavía un mayor asentamiento.

Otro tipo de actividades similares son las encomendadas a los personal librarians, una especie de tutores encargados del estudiante los primeros años. Se trata de una asistencia proactiva y personal fundamentalmente para trabajos académicos, pero es una opción no muy viable por la falta de recursos y poco operativa debido a las difusas necesidades de información de los alumnos en los primeros años de grado. 
Lo importante al margen de etiquetas es que en todos los perfiles citados el profesional bibliotecario adquiere un rol de "supervisor" o "tutor" en el proceso de aprendizaje. Sin embargo, si bien es cierto que en la actualidad los bibliotecarios trabajan en colaboración con los docentes para integrar programas de alfin en los planes de asignaturas, su presencia en las clases continua siendo un complemento (Li, 2012). Generalmente se limita a la impartición de clases sobre técnicas de búsqueda o recursos, incluyendo cómo evaluar la calidad y fiabilidad de la información.

Existen estudios sobre la eficacia de la integración bibliotecaria en la formación curricular, así Bowler y Street (2008) opinan que integrar elementos de alfin en las clases es menos eficaz que un acercamiento más explícito, en el que los bibliotecarios aparezcan claramente como "formadores". Otro modelo de colaboración es la presencia en los currículos de asignaturas completamente impartidas por bibliotecarios con el fin de "legitimar la contribución educativa de la biblioteca" (Owusu-Ansah, 2007). El bibliotecario-formador o alfabetizador informacional debe adaptarse al trabajo de las unidades de información, grupos o empresas, adquiriendo competencias didácticas y pedagógicas (Pinto-Molina; Uribe-Tirado, 2011).

El bibliotecario adquiere el rol de supervisor o tutor en el proceso de aprendizaje

La integración del bibliotecario en los planes de estudio crea una relación estudiante-bibliotecario muy beneficiosa (Hoffman, 2011). Mechaca (2012) apunta que esta relación ayuda a los estudiantes a tomar conciencia de lo que implica realizar una investigación y los prepara para evaluar y utilizar la información disponible, tanto tradicional como a través de internet, incluida la web social. Precisamente, hacer entender cómo evoluciona y cambia la naturaleza de la información en el escenario conectado que vivimos ha de ser uno de sus principales retos.

En todos los casos, un modelo de biblioteconomía integrada se crea de manera escalable, a medida que se forman las relaciones con la comunidad de aprendizaje. No existe un modelo único y válido para todas las instituciones, por lo que es difícil medir y establecer comparaciones sobre el modelo más apropiado para cada centro. Lo mismo sucede al tratar de clasificar las actividades realizadas por el bibliotecario integrado. Algunas de las más representativas en la bibliografía son las siguientes:

\section{Integración en un sistema de gestión de contenidos (CMS)}

El profesional puede proporcionar un servicio relacionado con el software educativo empleado (Blackboard, Moodle...). La integración en la operación del CMS favorece la interrelación del bibliotecario con la actividad docente. Muchas propuestas amplían su mera presencia como "participante en la asignatura" y le dan categoría de "profesor ayudante" (teaching assistant) cuyas funciones variarán dependiendo de la asignatura y de lo acordado con el profeso- rado. De esta manera el embedded librarian puede ofrecer materiales especializados (tutoriales, guías...) a lo largo del curso, y tener un mayor grado de participación construyendo relaciones con la clase, iniciando debates o resolviendo consultas a través del sistema.

Uno de los mayores retos es la definición del papel del bibliotecario en la gestión de cursos online. Veal y Bennett (2009) señalan que incluso en la misma institución su grado de integración variará en función de la asignatura. York y Vance (2009) consideran difuso su papel en asignaturas con pocas actividades de investigación personal por parte del estudiante. Evidentemente, una buena comunicación con el profesorado en la repartición de las tareas y el papel de la asignatura es la clave. Una de las mejores prácticas según Hoffman y Ramin (2010) es implicar a varios bibliotecarios desde el principio, lo cual contribuye a racionalizar los tiempos y evitar sobrecargas de trabajo.

Algunas posibilidades de integración que resume Becker (2010) son:

- Crear un sistema de "Pregunte al bibliotecario" (Ask a librarian) en forma de foro en alguno de los temas tratados en la asignatura o como elemento permanente. En algunos casos constituiría un servicio de referencia virtual.

- Creación y disposición de documentos para la adquisición de competencias informacionales (incluyendo tutoriales en forma de vídeo, guías de recursos temáticos como $\mathrm{Li}$ bguides...). Su situación ha de ser visible en el CMS.

- Ayuda y debates a través de diferentes foros. Es importante que el bibliotecario monitorice las respuestas, por ejemplo a través de sistemas de notificación o rss.

Esta presencia constante facilitaría la interacción y comunicación con los estudiantes, y empoderaría a los bibliotecarios universitarios en la comunidad, haciendo más evidente su presencia. El modelo es especialmente útil para alumnos a distancia ya que así alcanzan unas cuotas de interacción que de otra forma no tendrían. Buena parte de la bibliografía defiende un modelo más avanzado de integración para ellos, al necesitar un mayor contacto.

Sullo; Harrod; Butera; Gomes (2012) recomiendan integrar personal bibliotecario como apoyo en educación a distancia. Recalcan la planificación a priori de su labor, poniendo énfasis en lo visual y en la comunicación cara a cara (utilización de Skype o servicios como Elluminate live en Blackboard para la realización de tareas de referencia) y presencia continua de herramientas de apoyo como guías y tutoriales sobre citación, manejo de gestores bibliográficos...

Para evitar sobrecargas de trabajo Hoffman y Ramin (2010) proponen la creación de un "espacio abierto constante" en el CMS para estudiantes que funcione como link o punto de acceso central a los servicios bibliotecarios para alumnos y profesores. Sin embargo, esto no sustituiría la interacción personal con un bibliotecario integrado.

\section{Colaboración en el diseño de asignaturas o activida- des}

Los bibliotecarios integrados en la universidad comparten la misma misión institucional que los docentes: la educación. El programa educacional de la universidad ha de requerir la 
presencia curricular y actividades evaluables sobre habilidades informacionales del alumnado. Ser parte de la creación de un curso daría la oportunidad al bibliotecario de ser un supervisor del material docente, asegurándose su correcta citación, promoviendo de manera más efectiva la utilización de los recursos de la biblioteca, añadiendo lecturas complementarias...

Así, habría dos roles en la coordinación de las asignaturas: un experto en el contenido, el docente, y una vez terminado, se enviaría al bibliotecario, quien se aseguraría de los aspectos formales e informacionales. Para ello el bibliotecario ha de tener acceso al curso entero antes de su inicio para poder implicarse y enriquecer los materiales (Bozeman; Owens, 2008). Además al equipo bibliotecario se le ha de reconocer de manera oficial su papel en la coordinación de la asignatura, y debe asistir a las reuniones.

\section{Enseñanza compartida (presencial y online)}

Un paso más es la enseñanza de asignaturas, íntegras o compartidas: profesores y bibliotecarios son responsables de la preparación del curso, actividades, docencia y proceso de evaluación. Colaborar con los profesores en la creación de contenidos y en aspectos docentes acerca la biblioteca a los estudiantes, y va introduciendo habilidades informacionales en su día a día.

\section{Integración en redes sociales}

Las plataformas y herramientas sociales tienen un potencial pedagógico muy importante debido a sus múltiples posibilidades para la creación, reutilización y difusión de la información. Permiten nuevas formas de interacción de conocimiento en un entorno sin limitaciones espaciales o temporales. Luo (2010) considera que se ha de incluir la web social en las actividades de alfin, sin embargo, quizá no sea suficiente para que los estudiantes comprendan los cambios en la creación y transmisión de información que supone la web 2.0. Purdy (2010) recomienda a los docentes que pongan énfasis en las redes sociales desde la perspectiva de entornos flexibles de creación de conocimiento. Los bibliotecarios han de ser capaces de reorientar las actividades de alfin a la obtención de competencias acerca de la búsqueda y evaluación de información en la web social. EI perfil profesional del bibliotecario integrado puede tener en ellas, además, un aliado para incrementar su visibilidad en el curso colaborando en la creación de experiencias de aprendizaje más estimulantes.

Un caso interesante en los modelos de integración es el propuesto por Filgo (2011), quien relata la participación directa del bibliotecario a través de Twitter y de blogs. En el caso del primero se crearía para la clase un hashtag propio a través del cual se canalizaría la comunicación entre los alumnos y el profesional. En cada clase el profesor invita a los alumnos a establecer comunicación con éste, que permanece como un mediador virtual con la clase. Se producen de esta forma interesantes efectos de interacción y creación de conocimiento.

A la hora de enfrentarse con este tipo de actividades existen varios problemas a tener en cuenta. En primer lugar, la gestión de esta comunicación instantánea, que dependerá de las habilidades del bibliotecario. Por ejemplo Filgo relata cómo sus tweets enriquecían simultáneamente los contenidos que se estaban viendo en la clase en ese mismo instante con vínculos a bases de datos, Wikipedia, Youtube...

En este sentido, los blogs permiten una comunicación más pausada, ya que no requieren de inmediatez.

La monitorización de las respuestas también es un aspecto importante que el profesional ha de integrar en sus actividades. Para ello puede usar herramientas como The Old Reader para monitorizar blogs, o aplicaciones de escritorio como Tweetdeck, que permite gestionar varios perfiles sociales a la vez.

La flexibilidad y fluidez de estas plataformas sociales es un elemento a tener en cuenta de cara al diseño de programas de biblioteconomía integrada. Pueden crear relaciones de manera mucho más sencilla y acercar los recursos de información más rápido a la comunidad universitaria.

Colaborar con los profesores en la creación de contenidos y en aspectos docentes acerca la biblioteca a los estudiantes

\section{Conclusiones y propuestas}

Se ha ofrecido una visión general del concepto de "biblioteconomía integrada", identificando sus características básicas. Se constata una dificultad al delimitar este perfil profesional, debido a que esta actividad depende de los grupos a los que se sirve y de sus necesidades de información concretas, por lo que es complicado de manera institucional dar una respuesta igual a todas las necesidades de integración bibliotecaria en la comunidad académica.

No se trata sólo de que los bibliotecarios universitarios asuman funciones docentes sino de que se produzca una sinergia en equipos de trabajo y esto precisa cambios en las estructuras organizacionales de la universidad. Una institución con tendencia al desarrollo de servicios globales y centralizados como la universitaria, ¿cómo puede superar su rigidez estructural y establecer modelos colaborativos que impliquen la integración del personal bibliotecario en equipos y entornos especializados?

Aunque los beneficios educacionales de estos programas son evidentes, faltan todavía estudios que puedan medir el impacto real para poder desarrollar servicios realistas de acuerdo con la situación actual de la universidad. La integración de los bibliotecarios en las actividades curriculares, bien como partners, docentes o coordinadores de asignaturas, mantiene una imagen relevante del bibliotecario de cara a los usuarios. La mayoría de la bibliografía analizada se centra en su participación en cursos online a través de sistemas de gestión de aprendizaje. Por último, su ampliación a través de los nuevos medios sociales, ayuda a fortalecer relaciones con los estudiantes y los ayuda a enfrentarse de manera más sencilla y dinámica con sus necesidades de información. 


\section{Nota}

1. Sobre su traducción al español, aunque no esté estandarizado, se suele utilizar indistintamente "bibliotecario integrado" (Torres-Salinas, 2011), "incrustado" (Robinson, 2010), "embebido" (González-Fernández-Villavicencio et al., 2007).

\section{Bibliografía}

Becker, Bernd (2010). "Embebbed librarianship: a point-ofneed-service". Behavioral \& social sciences librarian, v. 2, n. 3, pp. 237-240.

http://dx.doi.org/10.1080/01639269.2010.498763

Bowler, Meagan; Street, Kori (2008). "Investigating the efficacy of embedment: experiments in information literacy integration". Reference services review, v. 36, n. 4, pp. 438449.

http://dx.doi.org/10.1108/00907320810920397

Bozeman, Dee; Owens, Rachel (2008) "Providing services to online students: embedded librarians and access to resources". Mississippi libraries, v. 72, n. 3, pp. 57-59.

Covone, Nicole; Lamm, Mia (2010). "Just be there: campus, department, classroom... and kitchen?". Public services quarterly, v. 6, n. 2-3, pp. 198-207.

http://dx.doi.org/10.1080/15228959.2010.498768

Dewey, Barbara I. (2004). "The embedded librarian: strategic campus collaborations". Resource sharing \& information networks, v. 17, n. 1-2, pp. 5-17.

http://dx.doi.org/10.1300/J121v17n01_02

Drewes, Kathy; Hoffman, Nadine (2010). "Academic embedded librarianship: an introduction". Public services quarterly, v. 6, n. 2/3, pp. 75-82.

http://dx.doi.org/10.1080/15228959.2010.498773

Filgo, Ellen H. (2011). “\#Hashtag librarian: embedding myself into a class via Twitter and blogs". Computers in libraries, v. 31, n. 6, pp. 78-80.

Giuse, Nunzia B. (1997). "Advancing the practice of clinical medical librarianship". Bulletin of the Medical Library Association, v. 85, n. 4, pp. 437-438.

http://www.ncbi.nlm.nih.gov/pmc/articles/PMC226305/ pdf/mlab00097-0127.pdf

González-Fernández-Villavicencio, Nieves; Sánchez-Baíllo, Paz; Valor-Piechotta, Magdalena (2007). "La biblioteca embebida" (póster). En: 10 Jornadas españolas de documentación, Fesabid. Santiago de Compostela.

http://www.slideshare.net/nievesglez/la-bibliotecaembebida-en-la-plataforma-virtual http://eprints.rclis.org/9551/1/La_Biblioteca_Embebida2. $p p t$

Hoffman, Starr (2011). "Embedded academic librarian experiences in online courses: roles, faculty collaboration, and opinion". Library management, v. 32, n. 6/7, pp. 444-456. http://dx.doi.org/10.1108/01435121111158583

Hoffman, Starr; Ramin, Lilly (2010). "Best practices for librarians embedded in online courses". Public services quarterly, v. 6, n. 2-3, pp. 292-305. http://dx.doi.org/10.1080/15228959.2010.497743

Kvenild, Cassandra; Calkins, Kaijsa (eds.) (2011). Embedded librarians: moving beyond one-shot instruction. Chicago, IL: Association of College and Research Libraries. ISBN: 9780 838985878

Li, Judy (2012). "Serving as an educator: a Southern case in embedded librarianship". Journal of business \& finance librarianship, v. 17, n. 2, pp. 133-152.

http://dx.doi.org/10.1080/08963568.2012.661198

Luo, Lili (2010). "Web 2.0 integration in information literacy instruction: an overview". The journal of academic librarianship, v. 36, n. 1, pp. 32-40.

http://dx.doi.org/10.1016/j.acalib.2009.11.004

Matava, Tobie; Coffey, Dan; Kushkowski, Jeffrey (2010). "Beyond library walls: embedding librarians in academic departments". Public services quarterly, v. 6, n. 2-3, pp. 165173.

http://dx.doi.org/10.1080/15228959.2010.497835

Mechaca, Frank (2012). "The future is in doubt: librarians, publishers, and networked learning in the $21^{\text {st }}$ century". Journal of library administration, v. 52, n. 5, pp. 396-410. http://dx.doi.org/10.1080/01930826.2012.700804

Owusu-Ansah, Edwuard K. (2007). "Beyond collaboration: seeking greater scope and centrality for library instruction". Libraries and the academy, v. 7, n. 4, pp. 415-429. http://dx.doi.org/10.1353/pla.2007.0043

Pinto-Molina, María (2011). "Formación alfin desde y para el crai: retos y prospectivas". http://www.sedic.es/CRAI-Maria_Pinto.pdf

Pinto-Molina, María; Uribe-Tirado, Alejandro (2011). “Formación del bibliotecario como alfabetizador informacional". Anuario ThinkEpi, v. 5, pp. 13-21.

http://eprints.rclis.org/15790

Purdy, James (2010). "The changing space of research: web 2.0 and the integration of research and writing environments". Computers and composition, v. 27, n.1, pp. 48-58. http://dx.doi.org/10.1016/j.compcom.2009.12.001

Robinson, Nicolás (2010). "El bibliotecario 'incrustado'". Docu ¿qué?: entre olas de información, marzo. http://entreolasdeinformacion.blogspot.com.es/2010/03/ el-bibliotecario-incrustado.html

Shumaker, David; Talley, Mary (2009). "Models of embedded librarianship: Final report". Special Libraries Association.

http://www.sla.org/pdfs/EmbeddedLibrarianshipFinalRptRev. $p d f$

Shumaker, David (2012). The embedded librarian: innovative strategies for taking knowledge where it's needed. New Jersey: Information Today. ISBN: 9781573874526

Sullo, Elaine; Harrod, Tom; Butera, Gisela; Gomes, Alexandra (2012). "Rethinking library service to distance education students: analyzing the embedded librarian model". Medical reference services quarterly, v. 31, n. 1, pp. 25-33.

http://dx.doi.org/10.1080/02763869.2012.641822 
Torres-Salinas, Daniel (2011). “Integrados en la investigación: los embedded librarians". Anuario ThinkEpi, v. 5, pp. 48-51. http://ec3.ugr.es/publicaciones/ThinkEPI-2011.pdf

Veal, Robin; Bennett, Erika (2009). "The virtual library liaison: a case study at an online university". Journal of library administration, v. 49, n. 1-2, pp. 161-170. http://dx.doi.org/10.1080/01930820802312938

York, Amy; Vance, Jason (2009). "Taking library instruction into the online classroom: best practices for embedded librarians". Journal of library administration, v. 49, n. 1-2, pp. 197-209.

http://dx.doi.org/10.1080/01930820802312995

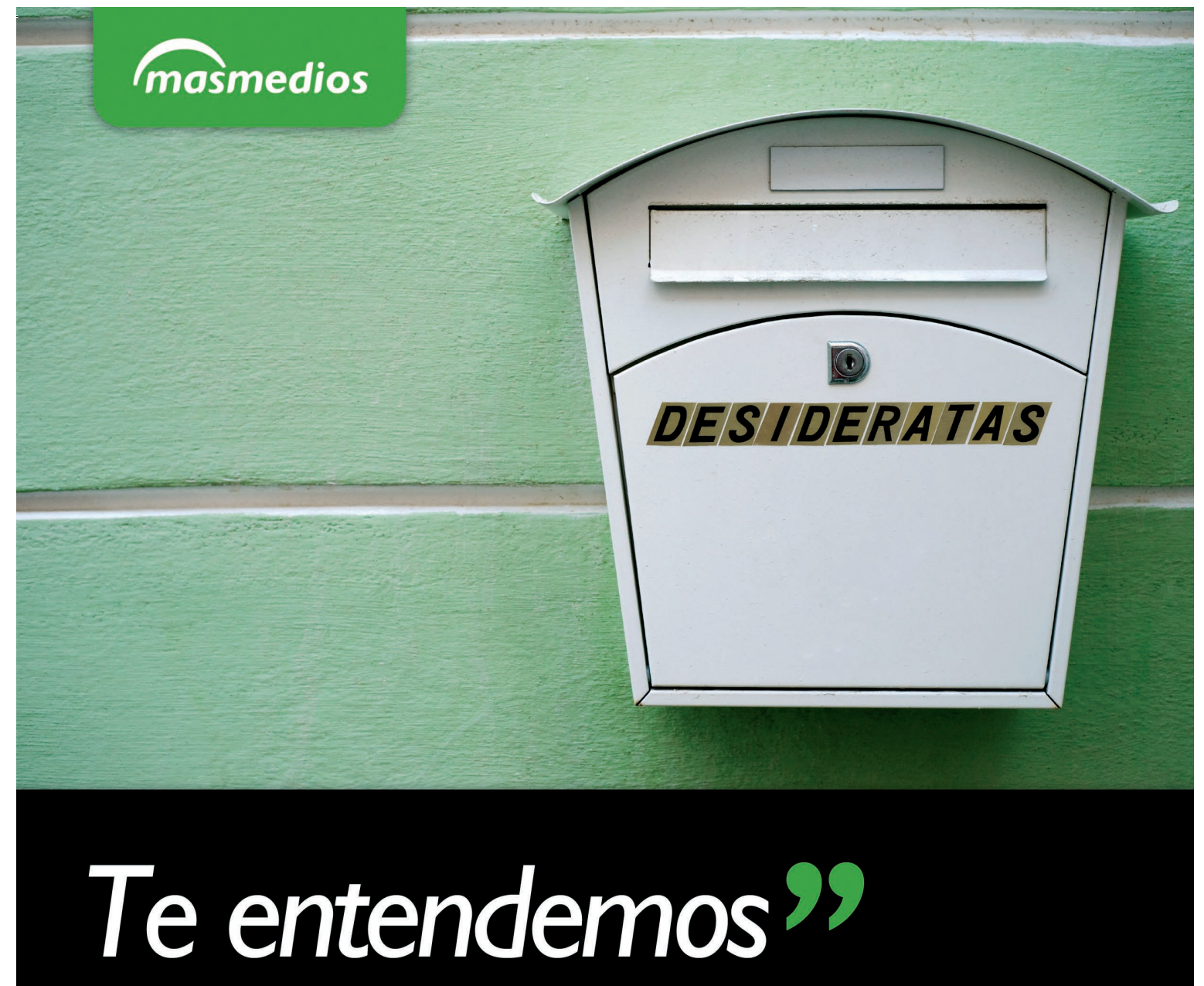

Sistemas de gestión de bibliotecas Open Source Interfaces interactivas y OPACs Repositorios OAI Gestión documental y de archivos Digitalización

Outsourcing de servicios documentales Desarrollo de sitios web / multimedia / e-learning Comunicación y e-marketing de servicios de información

$$
\begin{array}{lll}
\text { Oficinas Centrales: } & \text { Tel: } 96 \quad 3694123 \\
\text { mas } & \text { Fax: } 96 \quad 36934 \quad 39 \\
\text { C/Garcilaso I5-B } & \text { info@ @masmedios.com }
\end{array}
$$

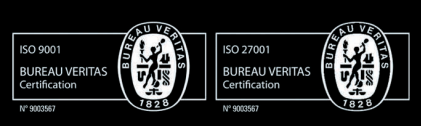

www. masmedios.com 